\title{
Human-biting activities of Anopheles species in south-central Ethiopia
}

Oljira Kenea ${ }^{1,2^{*}}$ D, Meshesha Balkew ${ }^{3}$, Habte Tekie ${ }^{1}$, Teshome Gebre-Michael ${ }^{3}$, Wakgari Deressa ${ }^{4}$, Eskindir Loha ${ }^{5}$, Bernt Lindtjørn ${ }^{6}$ and Hans J. Overgaard ${ }^{7,8,9}$

\begin{abstract}
Background: Indoor residual spraying (IRS) and long-lasting insecticidal nets (LLINS) are the key malaria vector control interventions in Ethiopia. The success of these interventions rely on their efficacy to repel or kill indoor feeding and resting mosquitoes. This study was undertaken to monitor human-biting patterns of Anopheles species in south-central Ethiopia.
\end{abstract}

Methods: Human-biting patterns of anophelines were monitored for 40 nights in three houses using human landing catches (HLC) both indoors and outdoors between July and November 2014, in Edo Kontola village, south-central Ethiopia. This time coincides with the major malaria transmission season in Ethiopia, which is usually between September and November. Adult mosquitoes were collected from 19:00 to 06:00 $\mathrm{h}$ and identified to species. Comparisons of HLC data were done using incidence rate ratio (IRR) calculated by negative binomial regression. The nocturnal biting activities of each Anopheles species was expressed as mean number of mosquitoes landing per person per hour. To assess malaria infections in Anopheles mosquitoes the presence of Plasmodium falciparum and $P$. vivax circumsporozoite proteins (CSP) were determined by enzyme-linked immunosorbent assay (ELISA).

Results: Altogether 3,408 adult female anophelines were collected, 2,610 (76.6\%) outdoors and 798 (23.4 \%) indoors. Anopheles zeimanni was the predominant species (66.5\%) followed by An. arabiensis (24.8\%), An. pharoensis (6.8 \%) and An. funestus (s.l.) (1.8\%).

The overall mean anopheline density was 3.3 times higher outdoors than indoors (65.3 vs 19.9/person/night, IRR: 3.3, $95 \%$ Cl: 1.1-5.1, P= 0.001). The mean density of An. zeimanni, An. pharoensis and An. funestus (s.l.) collected outdoors was significantly higher than indoors for each species $(P<0.05)$. However, the mean An. arabiensis density outdoors was similar to that indoors (11.8 vs 9.4/person/night, IRR: 1.3, $95 \%$ Cl: $0.8-1.9, P=0.335$ ). The mean hourly human-biting density of An. arabiensis was greater outdoors than indoors and peaked between 21: 00 and 22:00 h. However, An. arabiensis parous population showed high indoor man biting activities during bedtimes (22:00 to 05:00 h) when the local people were indoor and potentially protected by IRS and LLINs. All mosquito samples tested for CSP antigen were found negative to malaria parasites.

Conclusions: Results show much greater mosquito human-biting activities occurring outdoors than indoors and during early parts of the night, implying higher outdoor malaria transmission potential in the area. However, high bedtime (22:00 to 05:00 h) indoor biting activities of parous An. arabiensis suggest high potential intervention impact of IRS and LLINs on indoor malaria transmission.

Keywords: Malaria, Anopheles arabiensis, Endophagy, Exophagy, Ethiopia

\footnotetext{
* Correspondence: qabanef2015@gmail.com

${ }^{1}$ Department of Zoological Sciences, Addis Ababa University, Addis Ababa, Ethiopia

${ }^{2}$ Department of Biology, Wollega University, Nekemte, Ethiopia

Full list of author information is available at the end of the article
} 


\section{Background}

Malaria is the leading cause of death in wide parts of sub-Saharan Africa (SSA) [1]. Current malaria vector control in SSA relies heavily on indoor insecticidal interventions using indoor residual spraying (IRS) and longlasting insecticidal nets (LLINs) [1-3]. The scale up of IRS and LLINs during the last decade has substantially reduced malaria incidence in many parts of SSA $[4,5]$. These interventions reduce the density, feeding frequency and longevity of malaria vectors by killing the vectors with insecticides or blocking their contact with humans $[6,7]$ and primarily target malaria vectors that feed indoors and at night on sleeping humans [2].

However, following the adoption and scale-up of IRS and LLINs in SSA, a shift in mosquito behaviors has been observed, where mosquitoes more often bite humans outdoors and earlier in the evening, thereby avoiding insecticide treated surfaces and threatening the effectiveness of the interventions [8-11]. This behavioral change has been observed in Tanzania with An. arabiensis [8, 11]. On Bioko Island, Equatorial Guinea, high levels of outdoor biting by An. gambiae (s.s.) was observed throughout the night, including during early evening and morning hours when human hosts are often outdoors [9]. In Benin and Senegal, An. funestus has showed a behavioural change in biting activity after introduction of LLINs, remaining anthropophilic and endophilic, while adopting diurnal feeding when local people are not protected by IRS and LLINs [12, 13].

Nevertheless, in some countries in Africa, the principal vectors have shown consistent biting patterns and remain amenable to the effective IRS and LLINs interventions [14, 15]. For example, in Kenya, Bayoh et al. [15] found no evidence of behavioral shift of $A n$. gambiae (s.s.), An. arabiensis and An. funestusfrom the well-known pattern of late night, indoor biting characteristics of these typically highly anthropophilic species. Based on these results, it was recommended that malaria control interventions such as LLINs should continue to be prioritized [15].

In Ethiopia, $A n$. arabiensis, a member of $A n$. gambiae species complex, is the sole primary vector of malaria [16]. Other Anopheles species such as An. nili, An. funestus and $A n$. pharoensis are considered secondary vectors in the country [16]. Some evidence revealed that $A n$. arabiensis and $A n$. funestus were mainly endophagic and endophilic as compared to other Anopheles species such as $A n$. pharoensis, An. welcomi, An. zeimanni and $A n$. nili, which were mainly exophagic and exophilic [16-18]. Anopheles arabiensis were reported to bite indoors and outdoors throughout the night with peak activities at early part of the night before the inhabitants retire to bed [19-22]. This vector bites mainly on human and bovine hosts [16, 22-25]. Outdoor biting, early biting and biting on non-human hosts compromise the effectiveness of malaria vector interventions particularly IRS and LLINs that target endophagic, anthropophagic and nocturnal biting mosquitoes and worth to be monitored for evidence-based vector control interventions.

IRS has been used for more than four decades in Ethiopia [16, 26, 27]. Insecticide treated nets (ITNs) were introduced in 1997/1998 in selected malarious areas and the distribution of LLINs started in 2005 [27]. The most recent malaria strategic plan of the country is to achieve $100 \%$ LLIN ownership per sleeping space, and $90 \%$ IRS coverage by the end of 2015 [26]. Recently, malaria cases and deaths in Ethiopian hospitals have declined in conjunction with scale-up of malaria interventions with IRS, LLINs and artemisinin-based combination therapy (ACT) [27, 28]. Based on these successful interventions, Ethiopia is currently planning to eliminate malaria by 2020 and in light of this national target, monitoring the biting behaviour of Anopheles mosquitoes is crucial with respect to the efficacy of vector control interventions.

A cluster randomized trial investigating the effect of IRS and LLIN interventions combined or separate on disease outcomes has been carried out in Adami Tullu Jiddo Kombolcha district [29]. As part of this trial, the present study was undertaken to provide baseline data by monitoring local Anopheles species biting humans (anthropophagic anophelines) and their biting patterns in a village included in the trial.

\section{Methods}

\section{The study area}

This study was done in Edo Kontola village, Adami Tullu Jiddo Kombolcha district, south- central Ethiopia (Fig. 1). Edo Kontola is situated along Lake Zeway on the main road from Addis Ababa to Hawassa between Abosa and Batu towns. This village was selected based on past entomological studies [16] and recent pilot surveys [30] to study variations in biting patterns of anopheline species in the same locality and under similar environmental settings. The village is part of a clusterrandomized trial studying the effect of IRS and LLIN interventions during September 2014 and December 2016 [29]. The main environmental feature of the area is Lake Zeway which covers about $434 \mathrm{~km}^{2}$ area with average depth of $4 \mathrm{~m}$ [16]. The lake supports irrigation farms and fishing, the main economic activities in the district. The people usually cultivate rain-fed maize and other cereal crops during the rainy season (June to October) and mainly vegetables such as onions, tomatoes, potatoes, and green pepper by irrigation during the dry season (November to May) and the wet season as well. Many of the inhabitants of the village live in traditional African grass-thatched house locally known 


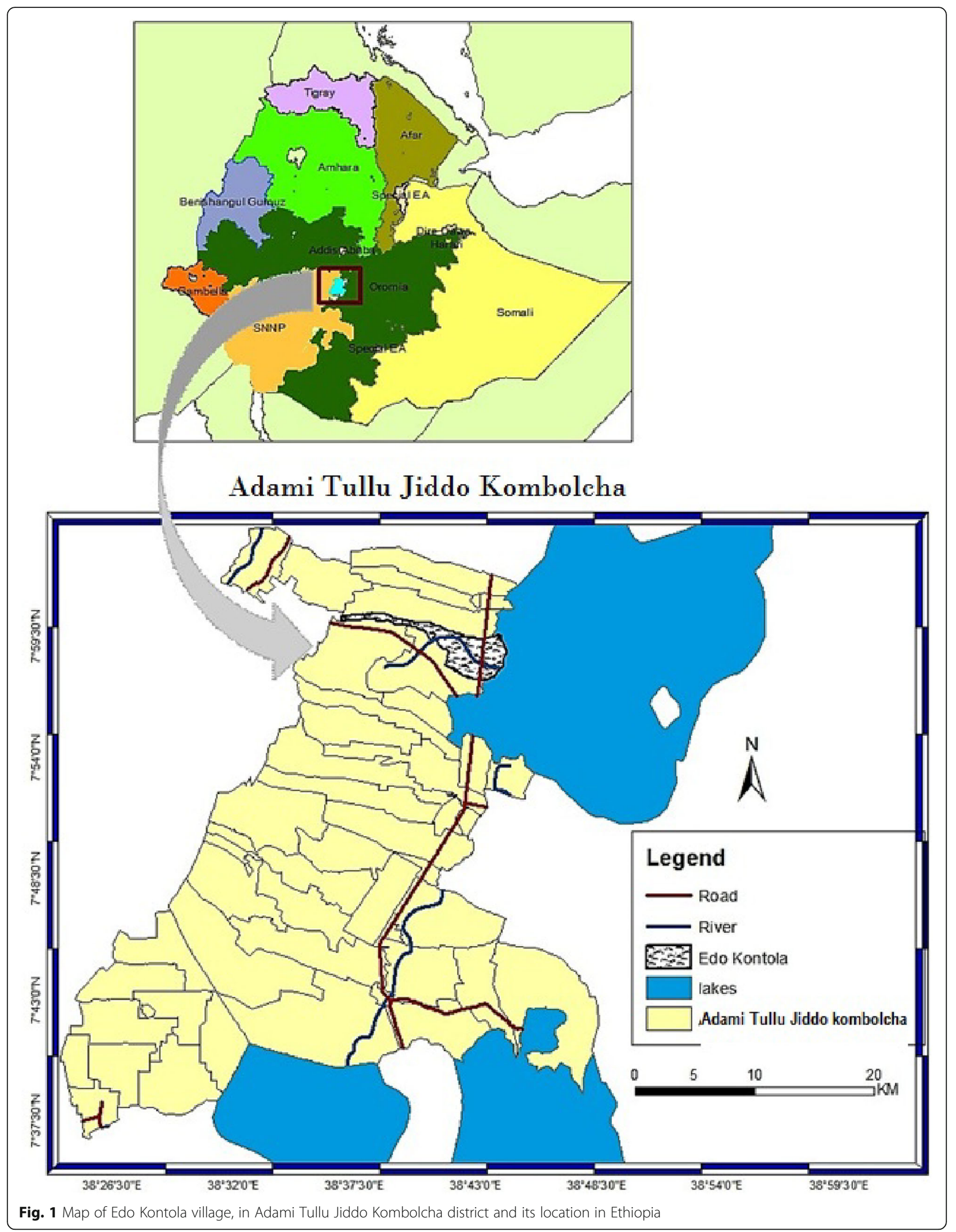


as 'mana chita' and some live in houses with corrugated iron roofs.

The lake area maintains malaria transmission by creating and sustaining potential mosquito breeding sites specifically at the lake shoreline [31]. Mosquito abundance increases as the lake fills and extends to the nearby villages during June to October and declines as the lake volume recedes during the following dry months. The area is semi-arid causing rain-fed surface pools in the uphill villages to dry up within a short time period. As a result, mosquito breeding sites are almost limited to the lake area even during the rainy season.

Malaria transmission is seasonal and driven by seasonal precipitation. The major malaria transmission season in Ethiopia is usually September to November. The most recent study in the district indicates that of 349 blood samples taken from febrile patients, 39 (11.2\%) slides were microscopically confirmed positive for malaria infection. The overall average malaria incidence was 4.6 per 10,000 person-weeks of observation. Higher malaria incidence was observed among children $<14$ years compared to older people and in villages near the lake shore than those distantly located from the lake [30]. The major malaria vector in the area is An. arabiensis whereas An. pharoensis plays a secondary role [16]. The recent pilot study results showed that An. arabiensis was susceptible to propoxur (carbamate), but resistant to pyrethroids. However, An. pharoensis was susceptible to all pyrethroids and carbamates tested [30]. The vectors transmit mainly Plasmodium vivax (85 \%) followed by $P$. falciparum (15\%) [30]. Malaria vector control relies on LLINs and IRS. In 2012, before the trial started, $27.3 \%$ of the households owned at least one LLINs and IRS was carried out in $91.7 \%$ of households in the district [30]. There is one health post in the village staffed by two health extension workers. The study village has approximately 35 households with 182 people inhabiting 17,500 square meters.

\section{Mosquito collections}

The nocturnal biting habits of Anopheles species were monitored for 40 nights using human landing catches (HLC) indoors and outdoors during July to November 2014, coinciding with the major malaria transmission season. The HLC, where human volunteers catch mosquitoes that land on their exposed body parts, was used because it is the gold standard method for monitoring mosquitoes that bite humans (anthropophagic mosquitoes) and the number of mosquitoes caught by HLC can directly provide an estimate of mosquito human-biting activities [32, 33].

Three houses close to the lakeshore were selected having similar size and design and with house owners agreeing to participate in the study. The houses were of traditional style with thatched conical-shaped roofs, circular floors and plastered walls. All houses had similar potential mosquito entry and exit points each having one door, eaves, and cracks in walls, but none of them had windows.

Each house in the village including the selected houses was located close to irrigation fields and within walking distance $(\leq 1 \mathrm{~km})$ from the lakeshore. It was also arranged in such way that the selected houses for HLC were free of cattle and human occupants on all collection nights. In addition, the houses were enrolled in the control arm of the trial and neither treated by IRS nor received LLINs during the study period [29].

The three houses were selected to reduce position bias driven by potential variations in indoor micro-climate such as indoor temperature, differences in mosquito entry points, mosquito density and proximity to animal shelter(s). Mosquito collections were performed in one house per night alternating each house for three consecutive nights per week. The collectors were rotated through the collection houses to compensate for any differences in attractiveness to mosquitoes and collecting abilities. Collections started in late July and ended in late November 2014 with intermittent collections in August and September. Mosquito collections were conducted by volunteers who were selected from the local people and who gave their written consent. Mosquitoes were collected from 19:00 to 06:00 h for 50 min each hour with 10 min rest for the volunteers. There were two collection shifts: one team of collectors worked from 19:00 to 24:00 $\mathrm{h}$ followed by the second team from 24:00 to 06:00 h. Every hour, two volunteers rotated between indoor and outdoor positions and carried out the work to reduce position bias. Outdoor collectors were positioned within $10 \mathrm{~m}$ from each study house. Each volunteer sat on a chair with the legs exposed from foot to knee and captured mosquitoes as soon as they land on the exposed legs before they commence feeding using a flashlight and mouth aspirator. Each hour's collection was kept separately in labeled paper cups. Supervisors were assigned to coordinate collection activities and watch volunteers not to fall sleep and bitten by mosquitoes over the study nights. The next morning, mosquitoes were identified to species by morphological characteristics using the standard identification key [34], and stored on silica gel for further analysis.

Molecular identification of sibling species was not done in this study; however, An. arabiensis was confirmed as the only member of An. gambiae species complex from previous studies and from our pilot study carried out from June to October, 2013 in the area [16, 30]. From the hourly collections, fresh and unfed An. arabiensis $(n=343)$ were selected for ovary dissection and determination of parity based on changes in the tracheoles of the ovaries under a microscope [35]. 
In order to determine the Plasmodium infection rate, the head and thorax of each mosquito $(n=2,560)$ were carefully separated from the abdomen and tested for the presence of $P$. falciparum and $P$. vivax circumsporozoite proteins (CSP) by the direct enzyme-linked immunosorbent assay (ELISA) [36].

\section{Estimation of entomological parameters}

Human-biting rates (HBRs) for each Anopheles species were calculated as mean number of mosquitoes collected by HLC per person per night $(\mathrm{m} / \mathrm{p} / \mathrm{n})$ separately for indoor and outdoor venues, i.e. $\mathrm{HBR}=$ no. of mosquitoes collected/no. of nights/no. of collectors [37]. The degree of endophagy was calculated as indoor $\mathrm{HBR}_{19: 00 \rightarrow 06: 00 \mathrm{~h} /}$ (indoor $\mathrm{HBR}_{19: 00 \rightarrow 06: 00 ~ \mathrm{~h}}+$ outdoor $\mathrm{HBR}_{19: 00 \rightarrow 06: 00 ~ h}$ ) while exophagy was calculated as outdoor $\mathrm{HBR}_{19: 00 \rightarrow 06: 00 \mathrm{~h}} /$ (outdoor $\mathrm{HBR}_{19: 00 \rightarrow 06: 00 ~} \mathrm{~h}+$ indoor $\mathrm{HBR}_{19: 00 \rightarrow 06: 00 \mathrm{~h}}$ ) [38].

The density of nocturnal biting was calculated as density of HBR during peak sleeping hours (hours starting 22:00 to 05:00) as follows [38]: (indoor $\mathrm{HBR}_{22: 00 \rightarrow 05: 00 \mathrm{~h}}$ + outdoor $\mathrm{HBR}_{22: 00 \rightarrow 05: 00} \mathrm{~h}$ )/(indoor $\mathrm{HBR}_{19: 00 \rightarrow 06: 00 ~} \mathrm{~h}+$ outdoor $\mathrm{HBR}_{19: 00 \rightarrow 06: 00 \mathrm{~h}}$ ). The nocturnal biting activities of each Anopheles species was expressed as mean number of each Anopheles species landing per person per hour separated by indoor and outdoor venues. Indoor and outdoor exposure to mosquito bites that took place early evening (19:00 to 22:00 h), during night 22:0005:00 h) and early in the morning (05:00-06:00 h) were estimated as the number of mosquito catches by HLC either indoors or outdoors divided by number of indoor and outdoor combined catches by each species multiplied by 100 . Parous rate was calculated as the total number of parous females for each species divided by the total number of mosquitoes dissected multiplied by 100 . The man biting proportions of parous An. arabiensis that took place during the early evening, during the night, and during the early morning (assessed by HLC) were compared based on field observations and available literature.

The ethical considerations for this study is described in more detail in the published protocol [29] and in the Declarations section below. In brief, to avoid the adverse effects of being bitten, mosquito collectors were trained to collect mosquitoes as soon the mosquitoes landed and before they bite. In order to minimize the risks, data collectors for the human landing catches were provided with an appropriate prophylactic drug (Malarone) before the commencement of sampling. To our knowledge, there are no reports on Malarone-resistant Plasmodium parasites in Ethiopia. The project provided blood examination and treatment of malaria free of charge for any study participant or householder who fell ill or wished to check himself. Fortunately, none of the mosquito collectors or householders were found parasite-positive during the study period.

\section{Meteorological data}

Meteorological data of the study area were obtained from the Meteorological Service Agency of Ethiopia, Addis Ababa.

\section{Data analysis}

Comparisons of indoor and outdoor HLC data were done by Generalized Linear Models (GLM) with negative binomial distribution. The impact of the collection venues on mean anopheline biting density were therefore estimated by exponentiation of negative binomial regression coefficient, i.e. Incidence Rate Ratio (IRR). Results were considered significant at $P<0.05$. Data were analyzed using the program SPSS version 20.0 (SPSS, Chicago, USA).

\section{Results}

Species composition and abundance of Anopheles mosquitoes

During the 40 nights of human landing collections, a total of 3,408 adult female anopheline mosquitoes were captured (Table 1). Anopheles zeimanni was the predominant species (66.5\%), followed by $A n$. arabiensis (24.8\%), An. pharoensis (6.8\%) and An. funestus (s.l.) (1.8\%). Overall, $76.6 \%(2,610)$ of the mosquitoes were captured outdoors and $24.4 \%$ (798) indoors.

\section{Human-biting rates}

The overall (indoor and outdoor combined) mean human-biting rate (HBR) of Anopheles mosquitoes was 85.2 mosquitoes/person/night $(\mathrm{m} / \mathrm{p} / \mathrm{n}$ ). The total (indoor and outdoor combined) mean HBRs for An. zeimanni was 56.7, An. arabiensis 21.1, An. pharoensis 5.8, and for An. funestus (s.l.) it was $1.6 \mathrm{~m} / \mathrm{p} / \mathrm{n}$.

The overall mean outdoor anopheline human-biting density (HBR) was 3.3 times higher than indoor (65.3 vs $19.9 \mathrm{~m} / \mathrm{p} / \mathrm{n}$, (IRR: 3.3, 95 \% CI: 1.1-5.1, $P<0.001$ ). The mean HBRs of An. zeimanni, An. pharoensis and $A n$. funestus (s.l.) collected outdoors were significantly higher than indoors for each species $(P<0.05$, Fig. 2). However, the mean outdoor HBR of An. arabiensis was similar to

Table 1 Total number and proportion of Anopheles species collected by human landing catches indoors and outdoors in Edo Kontola village, Ethiopia

\begin{tabular}{llll}
\hline Species & $\begin{array}{l}\text { Indoor } \\
n(\%)\end{array}$ & $\begin{array}{l}\text { Outdoor } \\
n(\%)\end{array}$ & $\begin{array}{l}\text { Total } \\
n(\%)\end{array}$ \\
\hline An. zeimanni & $351(15.5)$ & $1,916(84.5)$ & $2,267(66.5)$ \\
An. arabiensis & $375(44.4)$ & $470(55.6)$ & $845(24.8)$ \\
An. pharoensis & $50(21.5)$ & $183(78.5)$ & $233(6.8)$ \\
An. funestus (s.l.) & $22(34.9)$ & $41(65.1)$ & $63(1.8)$ \\
Overall & $798(23.4)$ & $2,610(76.6)$ & $3,408(100)$ \\
\hline
\end{tabular}




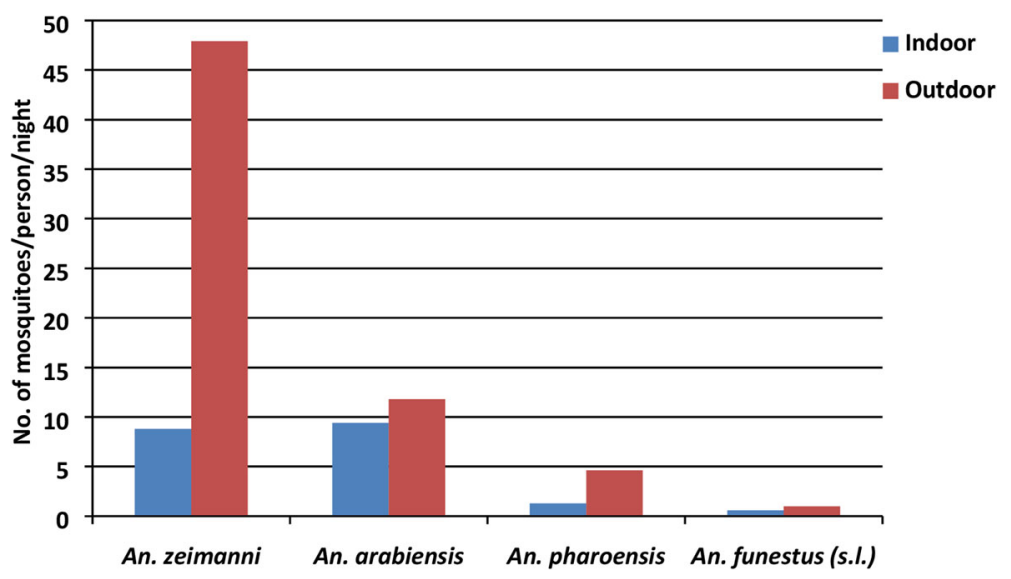

Fig. 2 Mean indoor and outdoor human-biting rates by Anopheles mosquitoes in central Ethiopia

that indoors $(11.8$ vs $9.4 \mathrm{~m} / \mathrm{p} / \mathrm{n}$, IRR: 1.3 , 95 \% CI: $0.8-$ 1.9, $P=0.335)$.

The mean HBR of An. zeimanni peaked in July and declined steeply until November (Fig. 3). However, mean HBR of An. arabiensis peaked in October and declined thereafter. HBRs of both An. pharoensis and An. funestus (s.l.) were low over the study months. Monthly precipitation in the area peaked in July, and fell to its lowest in November.

\section{Biting behaviours: endophagy, exophagy and nocturnality}

The degree of endophagy and exophagy (indoor and outdoor feeding) is given in Table 2. Overall, the majority of anophelines (76.6\%) exhibited exophagic (proportion of HBR outdoor) behaviour. The majority of An. zeimanni (84.5\%), An. pharoensis (79.3\%) and An. funestus (s.l.) (62.5\%) were captured outdoors and were clearly exophagic. For An. arabiensis, $55.7 \%$ and $44.3 \%$ of its population showed exophagic and endophagic behaviours, respectively.

With respect to nocturnality, overall, $48.2 \%$ of anopheline biting occurred during peak sleeping hours (22:00 to 05:00 h) as compared to when people were most likely awake (51.8\%). None of the Anopheles species showed marked peak nocturnality (high nocturnal biting activities during peak sleeping hours). Similar proportion of $A n$. arabiensis, An. pharoensis and An. funestus (s.l.) populations exhibited maximum human-biting activities during sleeping hours $(50.0 \%)$ when local people were potentially protected by LLINs and IRS as well as during non-sleeping hours (50.0\%) when the local people were not protected (Table 2).

\section{Human-biting patterns of anophelines and potential exposure to malaria mosquitoes}

The human-biting activity of $A n$. arabiensis was from dawn to dusk both outdoors and indoors with a single peak before midnight (21:00 to 22:00 h) outdoors followed by a general decline during the rest of the night. The indoor biting activity however showed two smaller peaks, one before midnight (20:00 to 21:00 h) and a second peak around midnight (24:00 to $01: 00 \mathrm{~h}$ ) (Fig. $4 \mathrm{a}$ ).

All the other anophelines were also active throughout the night, but with differing peak periods of biting activities both outdoors and indoors. The outdoor biting activities of both $A n$. pharoensis and An. zeimani were generally higher than indoors and both exhibited a pronounced unimodal biting activities early in the evening (19:00 to 20:00 h) which declined progressively during the rest of the night (Fig. 4b, c), while both species also followed the same pattern indoors, but with greatly reduced biting activities. On the other hand An. funestus (s.l.) appeared to show three peaks of biting activities outdoors of which two were the major ones: one before midnight (21:00 to 22:00 h) and another one early in the morning (5:00 to 6:00 h) between which was a smaller peak just after midnight (01:00 to 2:00 h) (Fig. 4d). The indoor biting activity on the other was bimodal with an early and smaller peak at 20:00 to 21:00 h and a major peak just before midnight (23:00 to 24:00 h). Humanbiting activities of the main malaria vectors; An. arabiensis and An. pharoensis peaked early in the evening (before 22:00 h) before local people retire to bed and were generally higher outdoors than indoors (Fig. 4a, b).

Altogether, $27.6 \%$ of the major malaria vector, $A n$. arabiensis bites took place during bedtime (22:00 to 05:00 h) and might be potentially prevented by LLINs alone whereas $44.4 \%$ of this vector bites could be prevented by LLINs + IRS during the study period (Table 3). However, only $7.6 \%$ of the potential secondary vectors, i.e. An. pharoensis, An. zeimanni and An. funestus (s.l.), bites occurred during bed time (22:00 to $05: 00 \mathrm{~h})$ and 


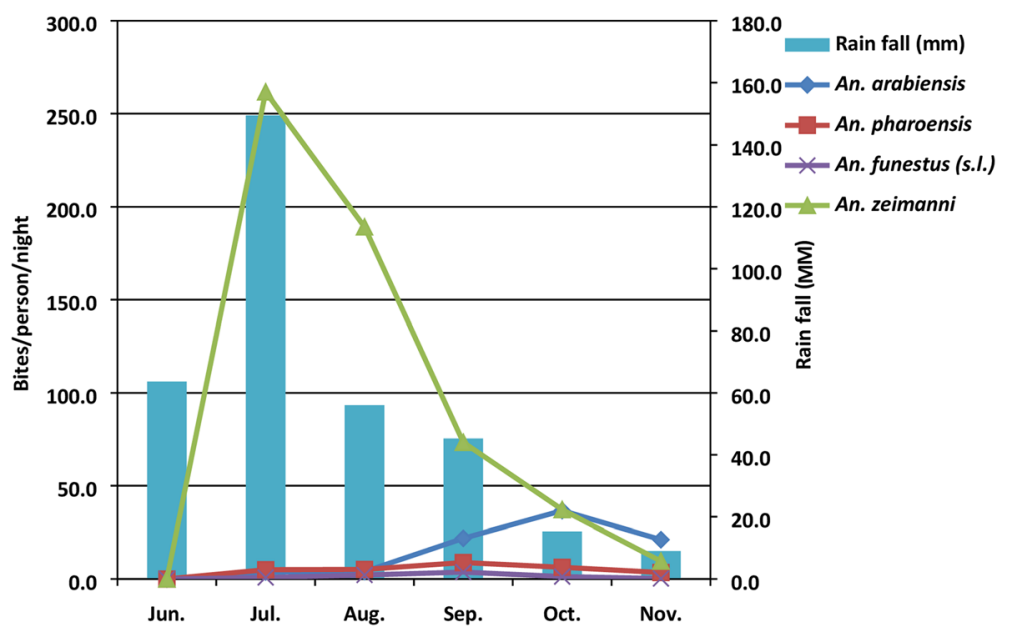

Fig. 3 Mean human-biting rates of Anopheles species and average precipitation for Edo Kontola village, Ethiopia

might be prevented by LLINs alone. Likewise, only $16.5 \%$ of these species bites might be prevented by IRS and LLINs combined interventions.

\section{Human-biting patterns of parous An. arabiensis population}

In total $343 \mathrm{An}$. arabiensis were dissected to determine parity rates and man biting patterns of the parous population. The overall indoor parous rate of An. arabiensis was $70.6 \%$ and the corresponding outdoor parous rate was $67.5 \%$ (Table 4). The proportion of parous $A n$. arabiensis population that showed indoor man biting activities during bedtimes (22:00 to 05:00 h) when the local people were indoors and potentially protected by IRS and LLINs was $72.4 \%$. Likewise $69.2 \%$ of parous An. arabiensis were collected while attempting to bite man before bedtimes (before 22:00 h). The proportion of parous $A n$. arabiensis (50.0\%) caught biting people during early morning (05:00 to 6:00 h) was low compared to either before bedtime or during bedtime. The overall indoor parous rate of $A n$. arabiensis was high (76.1\%) in October and low (54.5\%) in November. The corresponding outdoor parous rate was high $(75.0 \%)$ in September and low $(45.7 \%)$ in November. No ovarial dissection was carried out in July and August due to low mosquito density.

\section{Malaria infection}

A total of 1,500 An. zeimanni, 800 An. arabiensis, 200 An. pharoensis and 60 An. funestus (s.l.) were tested for the presence of CSP of $P$. falciparum and $P$. vivax. However, none was found positive. For this reason, the entomological inoculation rate (EIR) could not be determined.

\section{Discussion}

Anopheles zeimanni, An. arabiensis, An. pharoensis and An. funestus (s.l.) were found to be the four human-biting anopheline species occurring indoors and outdoors in the study area. This finding is similar to a recent pilot study [30] and previous entomological collections in the same area [16]. These results also showed that An. zeimanni (member of the An. coustani species complex) was the most predominant species and outnumbered the primary

Table 2 Human-biting rates (HBR; number of mosquitoes collected per person per night (95\% confidence interval), and feeding behaviors of Anopheles species in Edo Kontola village, Ethiopia

\begin{tabular}{|c|c|c|c|c|c|}
\hline Biting activities & An. arabiensis & An. pharoensis & An. zeimanni & An. funestus (s.l.) & Total \\
\hline Indoor HBR (19:00-06:00) & $9.4(7.9-11.0)$ & $1.2(0.8-1.7)$ & $8.8(6.1-11.6)$ & $0.6(0.1-1.1)$ & 20.0 \\
\hline Outdoor HBR (19:00-06:00) & $11.8(9.8-14.1)$ & $4.6(3.6-5.6)$ & $47.9(38.4-56.9)$ & $1.0(0.6-1.5)$ & 65.3 \\
\hline Nocturnal HBR (22:00-05:00) & $11.2(9.5-13.1)$ & $2.5(1.9-3.1)$ & $26.5(19.9-34.2)$ & $0.9(0.5-1.5)$ & 41.1 \\
\hline Endophagy (\%) & $44.3(43.8-44.6)$ & $20.7(18.2-23.3)$ & $15.5(13.7-16.9)$ & $37.5(14.3-42.3)$ & 23.4 \\
\hline Exophagy $(\%)^{b}$ & $55.7(55.4-56.2)$ & $79.3(76.7-81.8)$ & $84.5(83.1-86.3)$ & $62.5(57.7-85.7)$ & 76.6 \\
\hline Nocturnality $(\%)^{c}$ & $52.8(44.6-53.5)$ & $43.1(42.4-43.7)$ & $46.7(44.7-49.9)$ & $56.3(47.6-71.4)$ & 48.2 \\
\hline
\end{tabular}

a Proportion of indoor HBR between 19:00 and 06:00 $\mathrm{h}$

bProportion of outdoor HBR between 19:00 and 06:00 $\mathrm{h}$

${ }^{\mathrm{C}}$ Proportion of HBR between 22:00 and 05:00 $\mathrm{h}$ (during sleeping hours) 


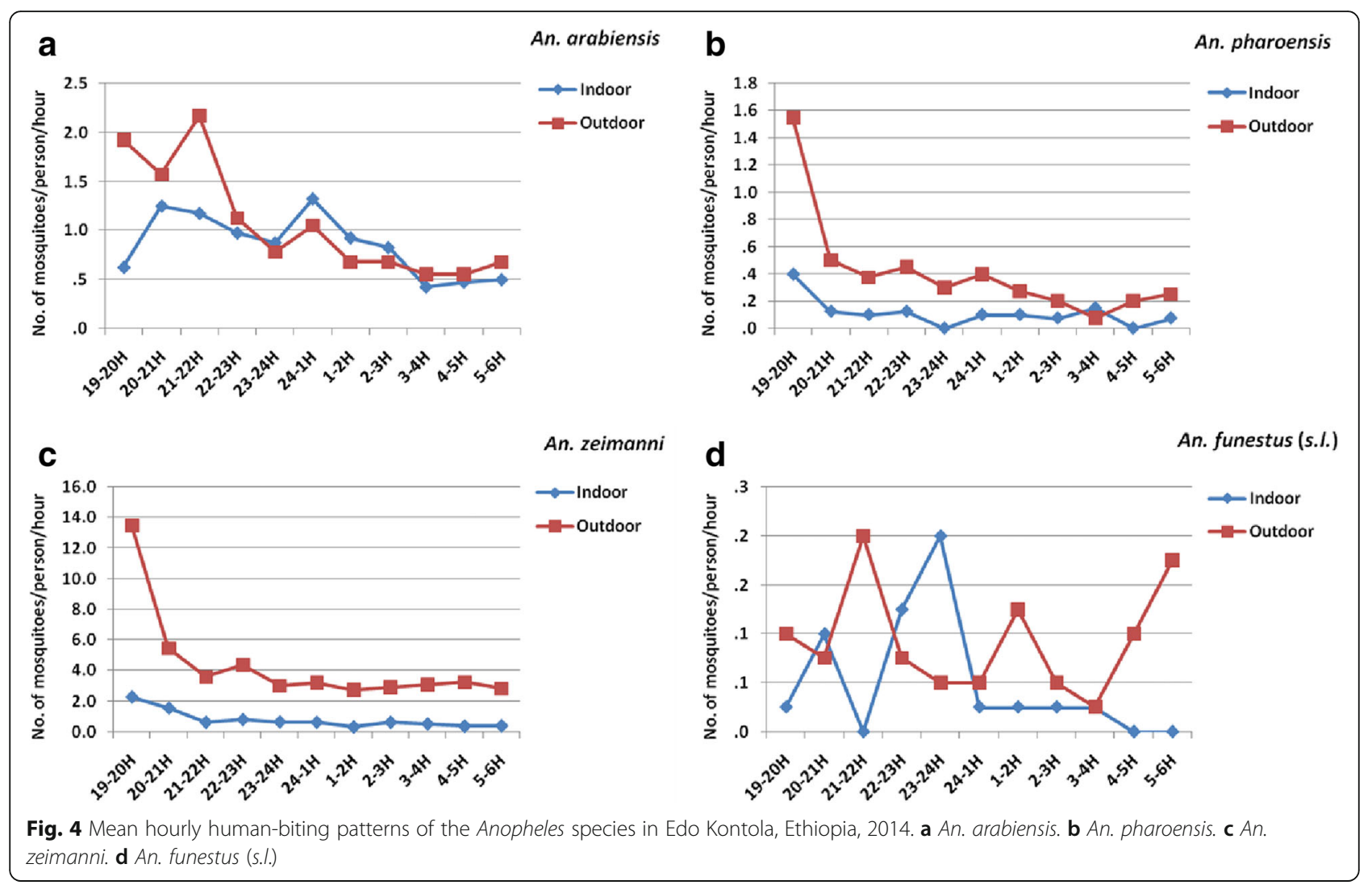

malaria vector, $A n$. arabiensis which was once the most abundant species [16]. Furthermore, An. arabiensis was the only member of An. gambiae complex [16, 30]. More recently, Bekele et al. [39] reported that An. zeimanni was among the most abundant anopheline species in three villages near the study village. The abundance of $A n$. zeimanni observed in the present study agrees with the earliest reports by Krafsur [17] who recorded that this species was the predominant species biting man near swamp margins in Gambela Region, western Ethiopia.

The abundance of An. zeimanni over An. arabiensis could be attributed to difference in their breeding site preferences. The present study village, Edo Kontola is located along Lake Zeway on the margin of swamp associated with aquatic vegetation that might have favored abundance of An. zeimanni that typically breed in vegetative swamps. Anopheles zeimanni has been found to be closely associated with aquatic vegetation [17] whereas $A n$. arabiensis typically breeds in small, sunlit temporary water pools [31]. Another potential reason could be inter species differences in feeding behaviours between An. zeimanni and An. arabiensis. Results showed that man-biting densities of An. zeimanni were significantly higher outdoor than indoor and exhibited more exophagic than endophagic behaviour unlike An. arabiensis which was a more endophagic species. This finding is consistent with previous reports in the country $[16,17]$. Because An. zeimanni was more exophagic than $A n$.

Table 3 Abundance of primary (An. arabiensis) and secondary [An. pharoensis, An. zeimanni, An. funestus (s.l.)] malaria vectors collected indoors and outdoors at different times of the night in Edo Kontola village, Ethiopia

\begin{tabular}{llllll}
\hline Anopheles species & Venue & $\begin{array}{l}\text { Early evening } \\
(19: 00-22: 00 \mathrm{~h})\end{array}$ & $\begin{array}{l}\text { Night } \\
(22: 00-05: 00 \mathrm{~h}) \\
n(\%)\end{array}$ & $\begin{array}{l}\text { Early morning } \\
(05: 00-06: 00 \mathrm{~h}) \\
n(\%)\end{array}$ & $\begin{array}{l}\text { Whole night } \\
(19: 00-06: 00 \mathrm{~h}) \\
n(\%)\end{array}$ \\
\hline Primary vector & & $n(\%)$ & $233(27.6)$ & $20(2.4)$ & $375(44.4)$ \\
& Indoor & $122(14.4)$ & $216(25.6)$ & $27(3.2)$ & $470(55.6)$ \\
Secondary vectors & Outdoor & $227(26.8)$ & $196(7.6)$ & $19(0.7)$ & $423(16.5)$ \\
& Indoor & $208(8.1)$ & $996(38.8)$ & $130(5.1)$ & $2,140(83.5)$ \\
\hline
\end{tabular}

Percentages were calculated as the number of mosquito catches by HLC, either indoor or outdoor, divided by the number of indoor and outdoor combined catches 
Table 4 Parity rates (in \%) of An. arabiensis (no. of parous/no. of tested) collected by human landing collections indoors and outdoors at different times of the night during three months in Edo Kontola village, Ethiopia

\begin{tabular}{|c|c|c|c|c|c|}
\hline \multirow[t]{2}{*}{ Month } & \multirow[t]{2}{*}{ Venue } & \multicolumn{3}{|l|}{ Time } & \multirow{2}{*}{$\begin{array}{l}\text { Total } \\
\text { Whole night } \\
\text { (19:00-06:00) }\end{array}$} \\
\hline & & $\begin{array}{l}\text { Early evening } \\
(19: 00-22: 00)\end{array}$ & $\begin{array}{l}\text { Night } \\
(22: 00-05: 00)\end{array}$ & $\begin{array}{l}\text { Early morning } \\
(05: 00-06: 00)\end{array}$ & \\
\hline \multirow[t]{2}{*}{ September } & Indoor & $58.3(7 / 12)$ & $69.2(9 / 13)$ & $0(0 / 0)$ & $72.7(16 / 22)$ \\
\hline & Outdoor & $40.0(2 / 5)$ & $84.6(11 / 13)$ & $100.0(2 / 2)$ & $75.0(15 / 20)$ \\
\hline \multirow[t]{2}{*}{ October } & Indoor & $77.8(28 / 36)$ & $76.0(38 / 50)$ & $50.0(1 / 2)$ & $76.1(67 / 88)$ \\
\hline & Outdoor & $71.8(56 / 78)$ & $73.3(44 / 60)$ & $57.1(4 / 7)$ & $71.7(104 / 145)$ \\
\hline \multirow[t]{2}{*}{ November } & Indoor & $50.0(10 / 20)$ & $61.5(8 / 13)$ & $0(0 / 0)$ & $54.5(18 / 33)$ \\
\hline & Outdoor & $62.5(10 / 16)$ & $33.3(6 / 18)$ & $0(0 / 1)$ & $45.7(16 / 35)$ \\
\hline \multirow[t]{2}{*}{ Total } & Indoor & $69.2(45 / 65)$ & $72.4(55 / 76)$ & $50.0(1 / 2)$ & $70.6(101 / 143)$ \\
\hline & Outdoor & $68.7(68 / 99)$ & $67.0(61 / 91)$ & $60.0(6 / 10)$ & $67.5(135 / 200)$ \\
\hline
\end{tabular}

arabiensis, it appears to have less chance to come in contact with LLINs and IRS. This might have favored $A n$. zeimanni, while $A n$. arabiensis population was affected by IRS and LLINs interventions. Although An. zeimanni has not been incriminated as a malaria vector in Ethiopia so far, it is a locally important vector in Cameroon [40]. In Ethiopia, a single malaria-infected An. zeimanni was observed in the western part of the country [41]. Its higher abundance and human-biting activities observed in the current study also imply that it has a potential role in malaria transmission. More studies are required on the infectivity, vectorial capacity and competence, ecology, and bionomics of $A n$. zeimanni.

The present results also revealed that the Anopheles mosquitoes, in general, bite more frequently outdoors than indoors. Because, both IRS and LLINs are indoorbased, high outdoor human-biting rates indicate possible outdoor malaria transmission potential in the area. These findings might compromise the efficacy and effectiveness of LLINs and IRS and point to the necessity of outdoor vector interventions. The results show evidence for the occurrence of residual malaria transmission potential but the magnitude and impact of such transmission warrants further investigation in the area and elsewhere in the country. Anopheles pharoensis exhibited more exophagic behaviour than endophagic behaviour. These results would be expected because, $A n$. pharoensis is an exophagic species in Ethiopia [16-18] and elsewhere in Africa [42].

Unlike the other anopheline species, there were no significant differences in outdoor and indoor humanbiting rates of $A n$. arabiensis. This indicates a high flexibility and plasticity of the vector with respect to indoor and outdoor feeding and potential host preferences. Previous studies show that $A n$. arabiensis bite both indoors and outdoors [16-18]. With respect to host preference, $A n$. arabiensis has shown opportunistic feeding behaviour in Ethiopia [25], exhibiting either anthropophagic
[23, 30, 43] or zoophagic behaviour [24]. This study did not look for host preferences because mosquito collections were done by HLC alone, which is an unsuitable method for blood meal source analysis.

Analysis of the biting patterns showed early-evening biting behaviour of $A n$. arabiensis with the highest peak occurring before 22:00 $\mathrm{h}$ indoors and outdoors at times when the local people are not protected by LLINs. We have observed that villagers, both children and adults, spend time outdoors performing various activities such as fishing, looking after their cattle and typically retire to bed after 22:00 h. Previous reports also indicated that the people retire to bed after 22:00 $\mathrm{h}$ [16]. These human activities can increase exposure to mosquito bites. Previous studies in the same study area $[16,20]$ and elsewhere in the country $[21,22]$ have also recorded early biting behaviour of $A n$. arabiensis. In contrast to the present results, some findings documented peak $A n$. arabiensis man-biting activities after 23:00 h [18]. In short, the previous and the present results suggest that An. arabiensis's behaviour is flexible and potentially opportunistic in terms of host preference, and feeding and resting habits [16, 18-25].

These flexible behaviours remain a key challenge for malaria control and elimination because the vector may be less vulnerable to IRS and LLINs, and as a result, may sustain malaria transmission. Although these behaviours are believed to be a consequence of long-term exposure to IRS and LLINs interventions in Ethiopia $[21,23]$, evidence is still lacking. Sufficient historical and up-to-date evidence about the impact of insecticidal interventions on $A n$. arabiensis population and behaviour is needed to suggest that the vector is showing behavioural adaptation or has consistent biting patterns in the country. These issues need special attention for malaria control and elimination efforts in the country.

The peak indoor and outdoor man-biting activities of An. pharoensis and An. zeimanni occurred during early 
hours of the evening and there has been no evidence of behavioural modifications or shifts. These results are in agreement with other studies undertaken in this area [16]. Anopheles funestus (s.l.) did not show clear indoor and outdoor human-biting patterns due to small numbers collected.

The overall indoor parity rate for An. arabiensis was $70.6 \%$ and is similar to earlier reports from the same area by Rishikesh [44] who recorded a constant parity rate ranging from $65-70 \%$ for An. gambiae (s.l.) presumably $A n$. arabiensis. With this parity rate, $A n$. arabiensis lived long enough to maintain indoor malaria transmission. Results show that indoor parity rates of $A n$. arabiensis were high at times when local people generally are asleep indoors and potentially under LLINs [16]. This implies that IRS and LLINs have high potential intervention impact on indoor malaria transmission.

Results also show that all mosquito samples tested by ELISA $(n=2,560)$ were negative for P. falciparum and $P$. vivax circumsporozoite protein infection. It is not uncommon to find sporozoite negative mosquito samples in areas with seasonal malaria transmission such as in this study area [16]. Low sporozoite infection rates have been repeatedly reported from the study area, for example, Rishikesh [44] found nine sporozoite-positive mosquitoes (0.2\%) out of 4,513 An. gambiae (s.l.) (An. arabiensis) dissected for salivary gland examination. Kibretet al. [20] also found $0.6 \%$ and $1.2 \%$ P. falciparum sporozoite rates among 509 An. pharoensis and 424 An. arabiensis, respectively, collected by CDC light traps and tested by ELISA in an irrigated village in the proximity of Zeway Lake. In contrast, no sporozoitepositive mosquitoes were detected in a non-irrigated village located relatively far from the lake [20]. The current malaria decline coinciding with the scale-up of vector interventions and malaria treatment measures in the country $[28,45]$ might have reduced malaria parasites in the mosquito population. Furthermore, it can be suggested that lack of large numbers of mosquito specimens due to low mosquito density in the area and lack of access to more sensitive sporozoite testing methods than ELISA (such as quantitative real-time PCR) to detect infective mosquitoes could be potential factors for the negative results.

\section{Conclusions}

Anopheles zeimanni, An. arabiensis, An. pharoensis and An. funestus (s.l.) were found to be the human-biting species in the area, all with outdoor biting behaviours. A high proportion of parous An. arabiensis were collected during night times, when the local people are usually indoors and potentially protected by IRS and LLINs. These results suggest that: (i) early and outdoor biting behaviour of $A n$. arabiensis could compromise the effectiveness of IRS and
LLINs and point to the need for complementary interventions, and (ii) IRS and LLINs still have an impact on indoor malaria transmission suggesting that application and adherence to these interventions need to be strengthened.

\section{Abbreviations}

HLC: Human landing catches of mosquitoes arriving to feed at humans; IRS: Indoor residual spraying of household walls with insecticides; LLINs: Long-lasting insecticide treated nets

\begin{abstract}
Acknowledgments
We are grateful to all of the human volunteers for their assistance in collecting mosquitoes by landing catches. We thank Mr. Wossen Sisay for technical assistance with field mosquito collection and processing.
\end{abstract}

\section{Funding}

This study was financially supported by grants from the Research Council of Norway (RCN) (Project number: 220554).

\section{Availability of data and material}

The datasets generated and/or analysed during the current study are available from the corresponding author on reasonable request.

\section{Author's contributions}

OK, MB, HT, TGM, WD, EL, BL and HJO conceived and designed the study. All involved in proposal writing and participated in field coordination, data collection, supervision and overall implementation of the study. OK analysed the data and drafted the manuscript. All authors read and approved the final manuscript.

\section{Competing interests}

The authors declare that they have no competing interests.

\section{Consent for publication}

Not applicable.

\section{Ethics approval and consent to participate}

Ethical approval for the study was obtained from the Institutional Review Board of the College of Health Sciences at Addis Ababa University, the Ministry of Science and Technology in Ethiopia (Ref: 3.10/446/06), and the Regional Committee for Medical and Health Research Ethics, (Ref: 2013/986/ REK Vest) Western Norway. The protocol for the trial was registered at the Pan African Clinical Trials Registry under the registration number PACTR201411000882128.

Verbal and written informed consent to take part in the study was obtained prior to the commencement of this study, from volunteers for landing catches who were older than 18 years of age and house owners using the local Afan Oromo language. For the human landing catches, a separate written informed consent describing the potential risks and benefits of the study was obtained from the volunteers. These volunteers were selected from the study village. The participants were instructed that involvement in the study was voluntary, and that they had the right to withdraw at any time regardless of reason. Assurance was also given that a refusal to participate in this study would not affect their access to services at the health posts in the study villages in the community.

\section{Author details}

${ }^{1}$ Department of Zoological Sciences, Addis Ababa University, Addis Ababa, Ethiopia. ${ }^{2}$ Department of Biology, Wollega University, Nekemte, Ethiopia. ${ }^{3}$ Akililu Lemma Institute of Pathobiology, Addis Ababa University, Addis Ababa, Ethiopia. ${ }^{4}$ Department of Preventive Medicine, School of Public Health, College of Health Sciences, Addis Ababa University, Addis Ababa, Ethiopia. ${ }^{5}$ School of Public and Environmental Health, Hawassa University, Hawassa, Ethiopia. ${ }^{6}$ Centre for International Health, University of Bergen, Bergen, Norway. ${ }^{7}$ Norwegian University of Life Sciences, Ås, Norway. ${ }^{8}$ Institut de Recherche pour le Développement (IRD), Maladies InfectieusesetVecteurs, Ecologie, Génétique, Evolution etContrôle (MIVEGEC), Montpellier, France. ${ }^{9}$ Department of Entomology, Faculty of Agriculture, Kasetsart University, Bangkok, Thailand. 
Received: 22 April 2016 Accepted: 22 September 2016

\section{Published online: 30 September 2016}

\section{References}

1. Dambach P, Traore I, Becker N, Kaiser A, Sie A, Sauerborn R. Ecologic malaria reduction for Africa-innovative tools for integrated malaria control. Glob Health Action. 2014;7:25908.

2. WHO. Control of residual malaria parasite transmission: Guidance note. Geneva: World Health Organization; 2014.

3. Braack L, Hunt R, Koekemoer LL, Gericke A, Munhenga G, Andrew D, et al. Biting behaviour of African malaria vectors: where do the main vector species bite on the human body? Parasit Vectors. 2015:8:76.

4. D'Acremont $V$, Lengeler $C$, Genton B. Reduction in the proportion of fevers associated with Plasmodium falciparum parasitaemia in Africa: a systematic review. Malar J. 2010;9:240.

5. Mharakurwa S, Mutambu SI, Mberikunashe J, Thuma PE, Moss WJ, Mason PR. Changes in the burden of malaria following scale up of malaria control interventions in Mutasa District, Zimbabwe. Malar J. 2013;12:223.

6. Mutuku FD, King CH, Mungai $P$, Mbogo C, Mwangangi J, Muchiri EM, et al. Impact of insecticide-treated bed nets on malaria transmission indices on the south coast of Kenya. Malar J. 2011;10:356.

7. Gimnig JE, Vulule JM, Lo TQ, Kamau L, Kolczak MS, Phillips-Howard PA, et al. Impact of permethrin-treated bed nets on the entomologic indicies in an area of intense year-round malaria transmission. Am J Trop Med Hyg. 2003;68:115-20.

8. Russell TL, Govella NJ, Azizi S, Drakeley CJ, Kachur SP, Killeen GF. Increased proportions of outdoor feeding among residual malaria vector populations following increased use of insecticide-treated nets in rural Tanzania. Malar J. 2011;10:80.

9. Overgaard HJ, Saebo S, Reddy MR, Reddy VP, Abaga S, Matias A, et al. Light traps fail to estimate reliable malaria mosquito biting rates on Bioko Island, Equatorial Guinea. Malar J. 2012;11:56.

10. Sokhna C, Ndiath MO, Rogier C. The changes in mosquito vector behaviour and the emerging resistance to insecticides will challenge the decline of malaria. Clin Microbiol Infect. 2013;19:902-7.

11. Kitau J, Boxborough RM, Tungu PK, Matowo J, Malima RC, Magesa SM, et al. Species shifts in the Anopheles gambiae complex: Do LLINs successfully control Anopheles arabiensis? PLoS one. 2012;7:e31481.

12. Moiroux N, Gomez MB, Pennetier C, Elanga E, Djènontin A, Chandre F, et al. Changes in Anopheles funestus biting behavior following universal coverage of long-lasting insecticidal nets in Benin. J Infect Dis. 2012;206:1622-29.

13. Sougoufara S, Diedhiou SM, Doucouré S, Diagne N, Sembène PM, Harry M, et al. Biting by Anopheles funestus in broad daylight after use of long-lasting insecticidal nets: a new challenge to malaria elimination. Malar J. 2014;13:125.

14. Killeen GF, Kihond J, Lyimo E, Oketch FR, Kotas ME, Mathenge E, et al. Quantifying behavioural interactions between humans and mosquitoes: evaluating the protective efficacy of insecticidal nets against malaria transmission in rural Tanzania. BMC Infect Dis. 2006;6:161.

15. Bayoh MN, Walker D, Kosgei J, Ombok M, Olang GB, Githeko AK, et al. Persistently high estimates of late night, indoor exposure to malaria vectors despite high coverage of insecticide treated nets. Parasit Vectors. 2014;7:380.

16. Abose T, Ye-Ebiyo Y, Olana D, Alamirew D, Beyene Y, Regassa L, et al. Reorientation and definition of the role of malaria vector control in Ethiopia; the epidemiology and control of malaria with special emphasis to the distribution, behavior and susceptibility to insecticides of anopheline vectors and chloroquine resistance in Ziway, Central Ethiopia and other areas. Addis Ababa: MOVBD, MOH Ethiopia;1998

17. Krafsur CS. The bionomics and relative prevalence of Anopheles species with respect to the transmission of Plasmodium to man in western Ethiopia. J Med Entomol. 1977;14:180-94.

18. Taye A, Hadis M, Adugna N, Tilahun D, Wirtz RA. Biting behaviour and Plasmodium infection rates of Anopheles arabiensis from Sille, Ethiopia. Acta Trop. 2006;97:50-4.

19. Yohannes M, Haile M, Ghebreyesus TA, Witten KH, Getachew A, Byass P, et al. Can source reduction of mosquito larval habitat reduce malaria transmission in Tigray, Ethiopia? Trop Med Inter Health. 2005;10:1274-85.

20. Kibret S, Petros B, Boelee E, Tekie H. Entomological studies on the impact of a small-scale irrigation scheme on malaria transmission around Zeway, Ethiopia. Ethiop J Dev Res. 2010;32:418-38.

21. Yohannes $M$, Boelee E. Early biting rhythm in the afro-tropical vector of malaria, Anopheles arabiensis, and challenges for its control in Ethiopia. Med Vet Entomol. 2012;26:103-5.
22. Taye B, Lelisa K, Emana D, Asale A, Yewhalaw D. Seasonal dynamics, longevity, and biting activity of anopheline mosquitoes in southwestern Ethiopia. J Insec Sci. 2016;16:1-7.

23. Tirados I, Costantini C, Gibson G, Torr SJ. Blood-feeding behaviour of the malarial mosquito Anopheles arabiensis: implications for vector control. Med Vet Entomol. 2006;20:425-37.

24. Massebo F, Balkew M, Gebre-Michael T, Lindtjørn B. Blood meal origins and insecticide susceptibility of Anopheles arabiensis from Chano in South-West Ethiopia. Parasit Vectors. 2013;6:44.

25. Animut A, Balkew M, Gebre-Michael T, Lindtjørn B. Blood meal sources and entomological inoculation rates of anophelines along a highland altitudinal transect in south-central Ethiopia. Malar J. 2013;12:76.

26. $\mathrm{FMOH}$. National malaria guidelines. 3rd edn. Addis Ababa: Addis Continental; 2012

27. Aregawi M, Lynch M, Bekele W, Kebede H, Jima D, Taffese HS, et al. Time series analysis of trends in malaria cases and deaths at hospitals and the effect of antimalarial interventions, 2001-2011, Ethiopia. PLoS One. 2014;9:e106359.

28. Otten M, Aregawi M, Werel W, Karema C, Medin A, Bekele W, et al. Initial evidence of reduction of malaria cases and deaths in Rwanda and Ethiopia due to rapid scale-up of malaria prevention and treatment. Malar J. 2009;8:14.

29. Deressa W, Loha E, Balkew M, Desalegne A, Gari T, Kenea O, et al. Combining long-lasting insecticidal nets and indoor residual spraying for malaria prevention in Ethiopia: study protocol for a cluster randomized controlled trial. Trials. 2016;17:20.

30. Gari T, Kenea O, Loha E, Deressa W, Hailu A, Balkew M, et al. Malaria incidence and entomological findings in an area targeted for a cluster randomized controlled trial to prevent malaria in Ethiopia: results from a pilot study. Malar J. 2016;15:145.

31. Kenea O, Balkew M, Gebre-Michael T. Environmental factors associated with larval habitats of anopheline mosquitoes (Diptera: Culicidae) in irrigated and major drainage areas in the middle course of the Rift Valley, central Ethiopia. J Vec B Dis. 2011:48:85-92.

32. Lima JBP, Rosa-Freitas MG, Rodovalho CM, Santos F, Oliveira RL. Is there an efficient trap or collection method for sampling Anopheles darlingi and other malaria vectors that can describe the essential parameters affecting transmission dynamics as effectively as human landing catches? - a review. Mem Inst Oswaldo Cruz. 2014;109:685-705.

33. Briët OJT, Huho B, Gimnig JE, Bayoh N, Seyoum A, Sikaala CH, et al. Applications and limitations of Centers for Disease Control and Prevention miniature light traps for measuring biting densities of African malaria vector populations: a pooledanalysis of 13 comparisons with human landing catches. Malar J. 2015;14:247.

34. Gillies MT, Coetzee M. A supplement to the Anophelinae of Africa South of the Sahara. Johannesberg: South African Institute of Medical Research; 1987.

35. Detinova TS. Age-grouping methods in diptera of medical importance with special reference to some vectors of malaria. Geneva: World Health Organization Monograph Series; 1962. p. 216.

36. Beier JPP, Wirtz RA, Whitmire RE, Mugambi M, Hockmeyer WT. Field evaluation of an enzyme-linked immunosorbent assay (ELISA) for Plasmodium falciparum sporozoite detection in anopheline mosquitoes from Kenya. Am J Trop Med Hyg. 1987;36:459-68.

37. Kabbale FG, Akol AM, Kaddu JB, Onapa AW. Biting patterns and seasonality of Anopheles gambiae sensu lato and Anopheles funestus mosquitoes in Kamuli District, Uganda. Parasit Vectors. 2013;6:340.

38. Govella NJ, Okumu FO, Killeen GF. Insecticide-treated nets can reduce malaria transmission by mosquitoes which feed outdoors. Am J Trop Med Hyg. 2010;82:415-9.

39. Bekele D, Belyhun Y, Petros B, Deressa W. Assessment of the effect of insecticide-treated nets and indoor residual spraying for malaria control in three rural kebeles of Adami Tullu District, South Central Ethiopia. Malar J. 2012;11:127.

40. Tabue RN, Nem T, Atangana J, Bigoga JD, Patchoke S, Tchouine F, et al. Anopheles ziemanni a locally important malaria vector in Ndop health district, north west region of Cameroon. Parasit Vectors. 2014;7:262.

41. Degefa T, Zeynudin A, Godesso A, Haile Michael Y, Eba K, Zemene E, et al. Malaria incidence and assessment of entomological indices among resettled communities in Ethiopia: a longitudinal study. Malar J. 2015;14:24

42. Antonio-Nkondjio C, Kerah C, Simard F, Awono-Ambene P, Chouaibou M, Tchuinkam T, et al. Complexity of malaria vectorial system in Cameroon: contribution of secondary vectors to malaria transmission. J Med Entomol. 2006;43:1215-21. 
43. Seyoum A, Balcha F, Balkew M, Ali A, Gebre-Michael T. Impact of cattle keeping on human biting rate of anopheline mosquitoes and malaria transmission around Zeway, Ethiopia. East Afr Med J. 2002;79:485-90.

44. Rishikesh N. Observations on anopheline vectors of malaria in an unsprayed Upland Valley in Ethiopia. Geneva: WHO/MAL/; 1966. 66.554.

45. Alemu A, Muluye D, Mihret M, Adugna M, Gebeyaw M. Ten year trend analysis of malaria prevalence in Kola Diba, North Gondar, Northwest Ethiopia. Parasit Vectors. 2012;5:173

Submit your next manuscript to BioMed Central and we will help you at every step:

- We accept pre-submission inquiries

- Our selector tool helps you to find the most relevant journal

- We provide round the clock customer support

- Convenient online submission

- Thorough peer review

- Inclusion in PubMed and all major indexing services

- Maximum visibility for your research

Submit your manuscript at www.biomedcentral.com/submit 